\title{
Evolution of infectious hematopoietic necrosis virus (IHNV), a fish rhabdovirus, in Europe over 20 years: implications for control
}

\author{
Peter-Joachim Enzmann ${ }^{1, *}$, Jeannette Castric ${ }^{2}$, Giuseppe Bovo ${ }^{3}$, Richard Thiery ${ }^{2,6}$, \\ Dieter Fichtner ${ }^{4}$, Heike Schütze ${ }^{4}$, Thomas Wahli ${ }^{5}$ \\ ${ }^{1}$ Friedrich-Loeffler-Institut, Federal Research Institute for Animal Health, Paul-Ehrlich-Str. 28, 72076 Tübingen, Germany \\ ${ }^{2}$ Afssa-site de Ploufragan/Brest, Technopôle Brest-Iroise, BP 70, 29280 Plouzané, France \\ ${ }^{3}$ Istituto Zooprofilattico Sperimentale delle Venezie, Viale dell'Università 10, 35020 Legnaro (PD), Italy \\ ${ }^{4}$ Friedrich-Loeffler-Institut, Federal Research Institute for Animal Health, Suedufer 10, 17493 Greifswald - Insel Riems, \\ Germany \\ ${ }^{5}$ National Fish Disease Laboratory, Centre for Fish and Wildlife Health, Institute of Animal Pathology, University of Bern, \\ POB 8466, 3001 Bern, Switzerland
}

${ }^{6}$ Present address: Afssa-site de Sophia-Antipolis, BP 111, 06902 Sophia-Antipolis, France

\begin{abstract}
The fish pathogenic rhabdovirus infectious hematopoietic necrosis virus (IHNV) causes substantial losses in European aquaculture. IHNV was first detected in Europe in 1987 and has since undergone considerable spread. Phylogenetic analyses of the full G-gene sequences of 73 isolates obtained from 4 countries in Europe (France, $\mathrm{n}=18$; Italy, 9; Switzerland, 4; Germany, 42) enable determination of the evolution of the virus in Europe since the first detection, and identification of characteristic changes within the G-genes of European strains. Further, the database allows us to analyse the pathways of distribution in Europe over time. The results suggest that in most of the recent cases, spread of IHNV was related to trade of infected fish. The data further demonstrate that knowledge of the sequence is required to determine the source of infections in farms.
\end{abstract}

KEY WORDS: IHNV evolution $\cdot$ Phylogenetic tree $\cdot$ Control

Resale or republication not permitted without written consent of the publisher

\section{INTRODUCTION}

Infectious hematopoietic necrosis virus (IHNV), the causative agent of infectious hematopoietic necrosis, belongs to the rhabdovirus genus Novirhabdovirus (Morzunov et al. 1995, Pringle 1999, Enzmann 2000, Fauquet et al. 2005). IHN was originally observed as a disease mainly of Pacific salmon and trout in enzootic areas in western North America. In 1987, IHNV was detected for the first time in Europe, in France and Italy (Baudin-Laurencin 1987, Bovo et al. 1987). In 1992, IHNV was isolated from rainbow trout Oncorhynchus mykiss in Germany, the same host species as in France and Italy (Enzmann et al. 1992). Since then, IHN has become a serious threat to the European rainbow trout farming industry, especially in various parts of France, Italy and Germany. The fish-farming industry in Europe is mostly private; there are only a few governmental hatcheries for the purpose of stocking wild waters. The capacity of the private fish farms varies from a few tons (t) $\mathrm{yr}^{-1}$ to $100 \mathrm{t}$ or more.

Prevention and control of infectious diseases in fish farms, as well as movements of live aquaculture animals between member states, are regulated in the European Union (EU; Council of the European Union 2006). IHN is a compulsorily notifiable disease. Dependent on the health status of aquaculture zones or compartments, 5 categories are defined: I, disease-free; II, surveillance programme; III, undetermined; IV, eradication programme; and V, infected. Fish may only be 
introduced into Category I farms from farms of Category I. The same is valid for Category II and IV farms. Category III farms may introduce fish from Category I, II and III farms. Category V farms may introduce fish from all categories. The status 'disease-free' is controlled every year by the competent authority. In the case of suspicion of IHN, samples are taken and examined in an authorised laboratory, the farms are placed under official surveillance and control measures are implemented to prevent spread of the disease. An epizootic investigation to detect the source of the infection and to determine whether fish have left the farm preceding the notification of the suspicion is initiated. In the case of confirmation of IHN, the farm is officially declared infected; a containment area is established, no restocking takes place and no fish are moved into, within or out of the containment area. Switzerland enacted laws corresponding to those of the EUmember states France, Italy and Germany. In particular, confirmation of infected fish in a farm results in immediate stamping out of the stocks.

For IHNV, 3 major virus genogroups designated U, $\mathrm{M}$ and $\mathrm{L}$, indicating their general correlation with the upper, middle and lower portions, respectively, of the IHNV geographical distribution in North America (Kurath et al. 2003) have been determined. Analyses of the European isolates revealed an affiliation to Genogroup M, and Enzmann et al. (2005) suggested that all isolates were progenies of a virus introduced into France and Italy in 1987. In the present study we report on the further distribution and development of IHNV in Europe by comparing the complete nucleotide sequences of the $\mathrm{G}$ genes from European isolates originating from France (18 isolates), Italy (9 isolates), Switzerland (4 isolates), and Germany (42 isolates).

\section{MATERIALS AND METHODS}

Virus isolates. Virus strains were obtained from national reference laboratories by the authors (France: J.C.; Italy: G.B.; Switzerland: T.W.; Germany: D.F.) or from German regional fish disease laboratories (Table 1). All IHNV isolates were obtained as virus in frozen cell-culture supernatants prepared in accordance with standardized fish health protocols from the EU (Commission of the European Communities 2001).

The origin and sequences of IHNV reference strains $\mathrm{RB}$, WRAC and SRCV, as well as isolates D332-92, Dfs62-95, Dfs42-95, Dfs30-95, Dfs13-98, Dfs8-99 and Dfr100-96 were described earlier (Enzmann et al. 2005). The new virus isolates used in the present study were not cloned in order to preserve natural quasispecies diversity. All analysed viral sequences, 73 in total, are summarized in Table 1. All virus isolates originated from fish farms rearing rainbow trout, except one (Dfs13-98), which was isolated from wild eel. Virus isolates were obtained after outbreaks of IHN or during routine inspections, in which cases samples from fish were taken.

RT-PCR and sequence analysis. RNA isolation and RT-PCR were performed as described previously (Enzmann et al. 2005). The primers IM2WS (5'-ACT ACT ATG CCC AGG AGA CA-3') and ILZA (5'-TTC CGC TGG AAG TCT CTC TT-3') were used for the amplification of a fragment from the end of the matrix protein gene $M$ to the start of the viral RNA polymerase gene L. The resulting RT-PCR products were 2299 nucleotides (nt) in length and comprised the region from nt 2780 to nt 5078, of the complete IHNV genome (GenBank accession no. X89213, Schütze et al. 1995). All sequences were confirmed by sequencing the PCR products in both orientations.

The phylogenetic studies were performed with the nucleotide sequence of the complete open reading frame (ORF) encoding for the IHNV glycoprotein G (nt 3007 to 4533; X89213, Schütze et al. 1995). For sequence alignments and phylogenetic analyses, the PAUP program (gcg, Wisconsin Package) was applied using bootstrap analysis with heuristic tree search and maximum parsimony. Bootstrap values exceeding $70 \%$ were considered to indicate significant relatedness. The complete nucleotide sequences of IHNV glycoprotein genes from European isolates presented here were submitted to the National Center for Biotechnology Information (NCBI) database. The respective GenBank accession numbers are listed in Table 1.

\section{RESULTS}

\section{IHNV isolates and phylogenetic tree}

To investigate the development of IHNV in Europe, we determined the nucleotide sequence of the $\mathrm{G}$ gene ORF from 73 European isolates originated from France, Italy, Switzerland and Germany between 1987 and 2007. Table 1 shows the list of viral isolates used in this study. These viruses were gained from outbreaks of IHN, or from routine examinations prescribed by EU legislation. All isolates represent different collection sites (one exception, see Subclade F). The evolution of European IHNV over the 20 yr period since the first isolation in Italy and France in 1987 is illustrated in the phylogenetic tree shown in Fig. 1; the results of this study are summarised in Table 2 . For comparative reasons, the North American reference strains WRAC, RB and SRCV, belonging to Genogroups $M, U$ and $L$, respectively, were included in this study. The nomen- 
Table 1. Infectious hematopoietic necrosis virus (IHNV). Virus isolates used in this study. 1FO: first French outbreak; 1 IO: first Italian outbreak; G ORF: G gene open reading frame. Prefixes: F, French isolates; CH, Swiss isolates; D, German isolates; I, Italian isolates. RB, WRAC and SRCV are reference isolates from North America

\begin{tabular}{|c|c|c|c|c|c|}
\hline Virus isolate & G ORF accession & Year of isolation & Virus isolate & G ORF accession & Year of isolation \\
\hline $1 \mathrm{FO}-87$ & X89213 & 1987 & Df1963-05 & EU676216 & $2005^{a, b}$ \\
\hline 1IO-87 & FJ711518 & 1987 & Dfr100-96 & EU676217 & 1996 \\
\hline F02-01 & EU331442 & 2001 & Dfr858-07 & EU676218 & $2007^{a}$ \\
\hline F92-02 & EU331443 & 2002 & Dfr864-03 & EU676219 & $2003^{\mathrm{a}}$ \\
\hline F003454-03 & EU331444 & 2003 & Dfr946-03 & EU676220 & 2003 \\
\hline F73801-04 & EU331445 & 2004 & Dfr951-05 & EU676221 & 2005 \\
\hline F28701-03 & EU331446 & 2003 & Dfr1013-05 & EU676222 & $2005^{\mathrm{a}}$ \\
\hline Fm13316-02 & EU331447 & 2002 & Dfr1019-03 & EU676223 & 2003 \\
\hline Fn6433-03 & EU331448 & 2003 & Dstgfv86-06 & EU676224 & $2006^{a, b}$ \\
\hline Fn11064-03 & EU331449 & 2003 & Dstgfv106-06 & EU676225 & 2006 \\
\hline Fn15209-04 & EU331450 & 2004 & Dswego-06 & EU676226 & $2006^{\mathrm{a}}$ \\
\hline Fn150974-04 & EU331451 & 2004 & Dw1973-05 & EU676227 & 2005 \\
\hline Fo43725-03 & EU331452 & 2003 & Dwb42-03 & EU676228 & 2003 \\
\hline Fp7136-04 & EU331453 & 2004 & Dfr3164-06 & EU676230 & $2006^{a}$ \\
\hline Fp7875-04 & EU331454 & 2004 & Dfr394-04 & EU676231 & $2004^{\mathrm{b}}$ \\
\hline Fp7946-04 & EU331455 & 2004 & Dfr47-96 & EU676232 & 1996 \\
\hline Fsa041874-04 & EU331456 & 2004 & Dns28-02 & EU676233 & 2002 \\
\hline F13849-05 & EU676229 & 2006 & Dnsfv234-07 & EU676234 & 2007 \\
\hline Fs360-06 & EU676237 & 2006 & Ds1831-05 & EU676235 & 2005 \\
\hline CH29-96 & EU676196 & 1996 & Ds2162-05 & EU676236 & 2005 \\
\hline CH118-02 & EU676197 & 2002 & I166-05 & FJ711510 & 2005 \\
\hline $\mathrm{CH} 247-00$ & EU676198 & 2000 & I208-06 & FJ711511 & 2006 \\
\hline СН308-97 & EU676199 & 1997 & I223-06 & FJ711512 & 2006 \\
\hline Dau26-97 & EU676200 & 1997 & I273-05 & FJ711513 & 2005 \\
\hline Dau28-97 & EU676201 & 1997 & I389-06 & FJ711514 & 2006 \\
\hline Dau32-97 & EU676202 & 1997 & I459-05 & FJ711515 & 2005 \\
\hline Dau37-97 & EU676203 & 1997 & I687-05 & FJ711516 & 2005 \\
\hline Dau55-98 & EU676204 & 1998 & I742-05 & FJ711517 & 2005 \\
\hline Dau64-98 & EU676205 & 1998 & D332-92 & AY331657 & 1992 \\
\hline Dau373-01 & EU676206 & 2001 & Dfs13-98 & AY331658 & 1998 \\
\hline Dau688-04 & EU676207 & 2004 & Dfs8-99 & AY331660 & 1999 \\
\hline Dau819-96 & EU676208 & 1996 & Dfs30-95 & AY331662 & 1995 \\
\hline Dau832-94 & EU676209 & 1994 & Dfs42-95 & AY331663 & 1995 \\
\hline Dau1036-96 & EU676210 & 1996 & Dfs62-95 & AY331664 & 1995 \\
\hline Dau1573-97 & EU676211 & 1997 & $\mathrm{RB}$ & Reference isolate & 1976 \\
\hline Dau1906-07 & EU676212 & 2007 & WRAC & Reference isolate & 1982 \\
\hline Dau2185-05 & EU676213 & $2005^{\mathrm{a}}$ & SRCV & Reference isolate & 1966 \\
\hline Dau4241-04 & EU676214 & 2004 & \multirow{2}{*}{\multicolumn{3}{|c|}{$\begin{array}{l}\text { a Virus transmission by infected fish } \\
{ }^{b} \text { Heterogeneity detected }\end{array}$}} \\
\hline De2305-05 & EU676215 & $2005^{a, b}$ & & & \\
\hline
\end{tabular}

clature of isolates is composed of an indicator for the European country of origin (F: France; I: Italy; $\mathrm{CH}$ : Switzerland; D: Germany), the specific identification number of the respective laboratory and the year of isolation following a hyphen.

Although not strongly supported (bootstrap value 67), Subclade F (Fig. 1) with the isolates from Dfs42-95 to Dfs30-95 is interesting because it represents only German strains isolated in the period from 1994 to 1997. Strains Dfs42-95 and Dfs62-95 are identical, but were obtained from 2 different farms in the same region (about $20 \mathrm{~km}$ apart). Strain Dfr47-96 persisted in one of these farms and was isolated in the following year; it differed from the original virus in $1 \mathrm{nt}$. Isolates with this genetic affiliation were never again detected in Germany after the application of radical measures in 1997. In these fish farms, farmers were not allowed to remove live fish after the first suspicion of infection, and stamping out and disinfection were effected after diagnosis; these measures were accompanied by controlling those farms to which fish were delivered from the diseased farm prior to the suspicion of infection, and effecting the same measures if virus transmission was verified.

Other groups clustered within the tree represent IHNV isolates from different European origins. The main sector from Dau2185-05 to I687-05 (Subclade A, Fig. 1) includes German and Italian isolates found from 1998 to 2007 . The first Italian isolate, 1IO-87, is basal to Subclade A, although with weak bootstrap support. 


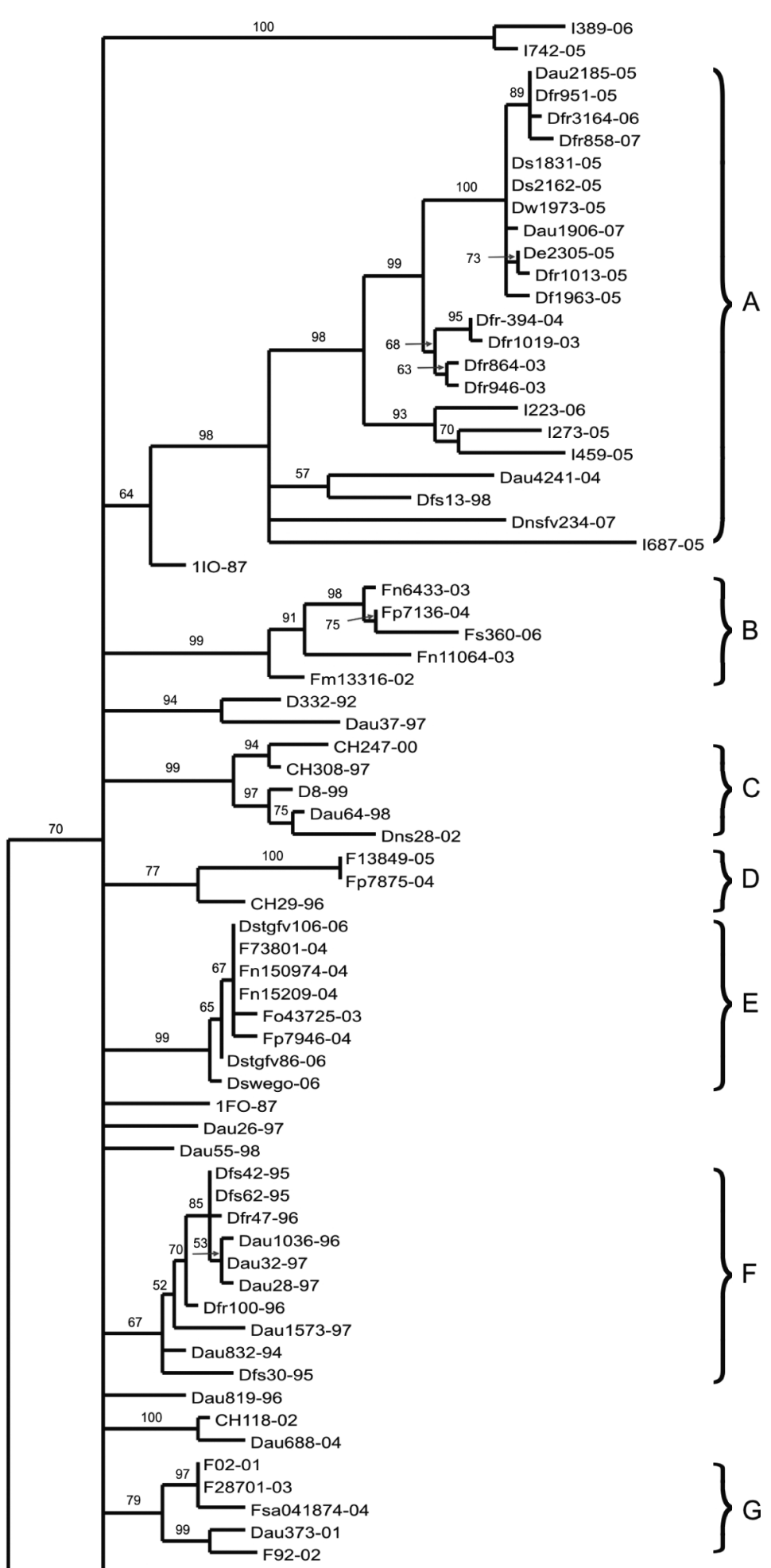

The year of isolation indicates that these strains are involved in the current circulation of IHNV in Germany. A more detailed analysis of transmission of IHNV between farms using the viruses circulating within a limited region in Germany is discussed later (Fig. 2). Adjacent to this group is a small subclade containing only French isolates detected from 2002 to 2006 (Fn6433-03 to Fm13316-02, Subclade B). The group from CH247-00 to Dns28-02 (Subclade C), observed between 1997 and 2002, demonstrates the distribution of a group of related viruses within Germany and Switzerland. The relation of Swiss IHNV to French isolates was demonstrated in the following cluster (Subclade D), in which the closely related French isolates F13849-05 and Fp7875-04 are grouped together with an older IHNV isolate detected in Switzerland in 1996.

In addition to the current situation of common viruses within Italy and Germany, France and Switzerland, and Germany and Switzerland, the following cluster (Subclade E, Fig. 1) contains a group of IHNV isolates circulating from 2003 until 2006 in France and Germany (Dstgfv106-06 to Dswego-06). Similarly, another subgroup (Subclade G), containing 5 viruses isolated from France and Germany in the years 2001 to 2004 with a bootstrap value of 79 is defined. From the summary of these results in Table 2, it is visible that Subclade A contains viruses actually circulating in Germany, whereas Subclade F viruses disappeared; further, a remarkable trend in crossing country boundaries is notable.

\section{Pathway of distribution of an IHNV-subgroup}

A detailed phylogenetic analysis of the events during circulation of a specific virus type within several independent private farms in the southern part of Germany is given in Fig. 2. This tree is part of Subclade A in Fig. 1. In Subclade A, a further subgroup (bootstrap value 100) comprises the German isolates Dau2185-05 to Dfr1963-05, which played a major role in an epizootic within a limited region in Germany. The geographic distribution of the viruses is shown in Fig. 3. Within this group, the

Fig. 1. Phylogenetic tree showing the evolution of infectious hematopoietic necrosis virus (IHNV) within about $20 \mathrm{yr}$ of circulation in Europe. 1FO: first French outbreak; 1IO: first Italian outbreak; Prefixes: F, French isolates; CH, Swiss isolates; D, German isolates; I, Italian isolates. RB, WRAC and SRCV are reference isolates from North America used as the outgroup; sequences were not delivered to GenBank. Bootstrap values exceeding $70 \%$ were considered to indicate significant relatedness. A-G: subclades 
Table 2. Infectious hematopoietic necrosis virus (IHNV). Summary of the phylogenetic study. F: France; CH: Switzerland; D: Germany; I: Italy

\begin{tabular}{|c|c|c|c|c|}
\hline Subclade & Bootstrap (\%) & No. of isolates & s Country & Years of isolation \\
\hline A & 98 & 22 & $\mathrm{D}, \mathrm{I}$ & $1998,2003-2007$ \\
\hline B & 99 & 5 & F & $2002,2003,2006$ \\
\hline $\mathrm{C}$ & 99 & 5 & $\mathrm{D}, \mathrm{CH}$ & 1997-2000, 2002 \\
\hline $\mathrm{D}$ & 77 & 2 & $\mathrm{~F}$ & 2004, 2005 \\
\hline $\mathrm{E}$ & 99 & 8 & $\mathrm{D}, \mathrm{F}$ & 2003, 2004, 2006 \\
\hline $\mathrm{F}^{\mathrm{a}}$ & $67^{\mathrm{b}}$ & 10 & $\mathrm{D}$ & 1995-1997 \\
\hline G & 79 & 5 & $\mathrm{~F}, \mathrm{D}$ & 2001-2004 \\
\hline \multicolumn{2}{|c|}{ Not clustered } & 20 & $\mathrm{D}, \mathrm{F}, \mathrm{I}, \mathrm{CH}$ & $\begin{array}{l}\text { 1987, 1992, 1996-1998, } \\
2001-2006\end{array}$ \\
\hline
\end{tabular}

Ds1831-05, Ds2162-05 and Dw were found. Fish trade records of these transfers could not be documented unequivocally (dashed lines in Fig. 3). The latter 3 farms are in the same region, located about $10 \mathrm{~km}$ apart from each other. The 3 viruses isolated from these 3 farms are identical but differ from the original virus (Dfr951-05) by 2 mutations (nt 199 and nt 1513). Virus Dw1973-05 was transmitted to 2 additional farms resulting in viruses De2305-05 (another farm belonging to the same owner) and Df1963-05, differing in 1 and 2 nt, respectively. Virus De2305-05 was further transmitted to a farm in which virus Dfr1013-05 was isolated, differing in 1 nt (Fig. 3).

\section{DISCUSSION}

In contrast to the previously used principle to clone all virus strains by at least 4 end-point titrations before sequencing (Enzmann et al. 2005), the new virus isolates sequenced for the present study were not cloned in order to detect quasi-species variation. The computed sequences were checked manually. In several consensus sequences a particular nt-peak was revealed to be a double peak, i.e. a smaller peak was located underneath the main peak (with corresponding occurrence in the complementary DNA strand). For these cases, the following interpretation was adopted: when the consensus sequence differed from a known sequence exactly in this particular nt, the consensus sequence was defined as a new virus strain descended from the known strain. The sequence of the original strain is hidden in the computed consensus sequence, because the field isolate is a mixture of the known virus strain and a mutated strain with the mutated strain in the majority. Thus, the ancestry of a new virus strain is determined (marked in Table 1 by superscript ' $b$ '). Using this method, current trends in the development of new virus strains can be discov-
Fig. 2. Phylogenetic tree demonstrating the circulation of specific infectious hematopoietic necrosis virus (IHNV) strains within the southern part of Germany. The relationship of the virus isolates is shown by arrows and the numbers of differing nucleotides (nt)

infection cycle could be partially iden-
tified and transfer of virus documented starting from isolate Dfr95105 . This virus was transmitted from the
first farm to 3 other farms. From these 3 farms, the following viruses could be isolated: Dau2185-05, which was iden-
tical to Dfr951-05 in the same year, 2005, and Dfr3164-06 isolated in 2006, which showed a single mutation, and mutations (Figs. 2 \& 3). In the same year in which Dfr951-05 was isolated, the virus was also transmitted to 3 additional farms in which viruses (1)

.




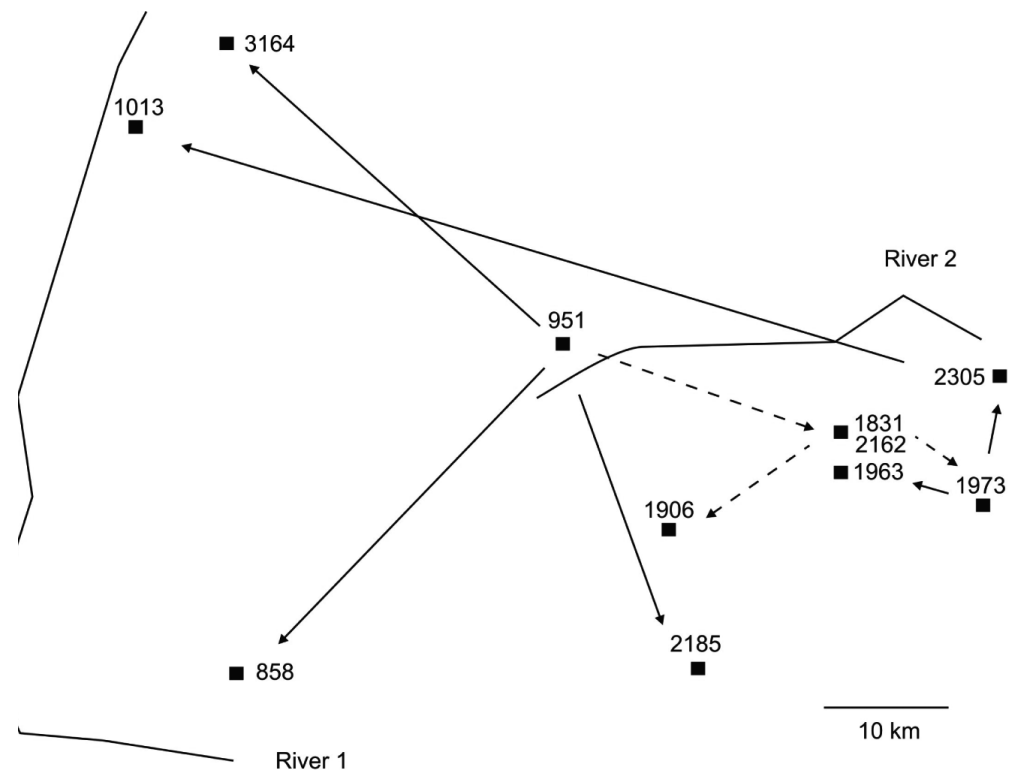

Fig. 3. Infectious hematopoietic necrosis virus (IHNV). Outline of the geographical relationship of outbreaks analysed in Fig. 2. Numbers represent virus isolates. Lines without arrows indicate rivers. Arrows indicate direction of transfer. Dashed lines mean that fish transport records were not documented unequivocally

ered at an earlier stage than with previously used methods. This also reveals that if field virus isolates are cloned after isolation, information on the development of the virus may be lost.

After 2003, intensive studies on the epidemiology and phylogeny of IHNV were initiated in Germany. The results of these analyses revealed that in the majority of IHN outbreaks the transmission of virus had occurred by trade of infected fish. This can also be concluded from the clustering pattern of German strains isolated after 2003 and is further supported by the fact that new outbreaks occurred after partial restocking of farms. It was possible in almost all cases to identify the origin of IHNV and the means by which it was introduced into a fish farm, and factors other than trade could be involved, e.g. transmission by birds. It was clearly demonstrated that IHNV travels through Europe apparently without significant restrictions. Closely related viruses found in Germany and France, in Germany and Switzerland, in France and Switzerland, as well as in Italy give evidence for this viral 'tourism' (Fig. 1, Table 2). The precise method of crossing borders remains to be determined by fish health officials. European regulations on the control of fish and fish egg transportation are clearly defined (Council of the European Union 2006), but nevertheless, the vast majority of fish farming in central Europe is private and violations cannot be excluded. The reason for frequent fish transport between private farms is governed by the law of supply and demand. Even small differences between prices can give rise to fish trade between private farms.

Phylogenetic analysis is revealed to be the most effective method of elucidating the ways of dissemination and evolution of IHNV and the correlation of virus distribution with trade channels. However, it could also be clearly demonstrated (Subclade F, Fig. 1) that strict sanitary measures eliminate IHNV, since after the application of rigorous measures in1997, isolates with this specific genetic makeup were never again detected in Germany.

From this study, it can be further concluded that development of IHNV may be very rapid in some cases. Within 1 year (2005), up to 4 mutations within the glycoprotein gene of IHNV occurred during the infection cycle in a limited region (Dfr951-05 and Df1963-05, Fig. 2), whereas in other cases, 1 (Dfr3164-06), 2 (Dfr858-07) and 3 (Dau1906-07) mutations occurred within 1 and 2 years.

The main risk of IHN outbreaks is the introduction of infected fish into a fish farm. Based on the guidelines of the World Organisation for Animal Health (OIE) and the EU (Council of the European Union 2006), IHN is a notifiable disease. In the EU, control measures are based on the isolation of the causal agent followed by serological identification. However, so far only sequence analyses of regions of the viral genome offer the capability of identifying and differentiating the isolates, thus providing a powerful tool in epidemiological investigations. Therefore, it is recommended that all newly detected IHNV isolates should be sequenced. Direct sequencing analyses of RT-PCR products provide the necessary information to identify new trends in the development of virus strains.

Acknowledgements. We thank A. Braun for excellent technical assistance.

\section{LITERATURE CITED}

Commission of the European Communities (2001) Commission Decision (2001/183/EC) 22 February 2001: laying down the sampling plans and diagnostic methods for the detection and confirmation of certain fish diseases and repealing Decision 92/532/EEC. Off J Eur Comm 44(LO67):65-76

Council of the European Union (2006) Council Directive (2006/88/EC) of 24 October 2006 on animal health requirements for aquaculture animals and products thereof, and on the prevention and control of certain diseases in aquatic animals. Off J Eur Union 49(L328):14-56 
Baudin-Laurencin F (1987) IHN in France. Bull Eur Assoc Fish Pathol 7:104

Bovo G, Giorgetti G, Jørgensen PEV, Olesen NJ (1987) Infectious haematopoietic necrosis: first detection in Italy. Bull Eur Assoc Fish Pathol 7:124

Enzmann PJ (2000) Molecular biology of fish pathogenic rhabdoviruses. In: Fingerman M, Nagabhushanam R (eds) Recent advances in marine biotechnology, Vol 5. Immunobiology and pathobiology. Science Publishers, Enfield, p 269-293

Enzmann PJ, Dangschat H, Feneis B, Schmitt D, Wizigmann G, Schlotfeldt HJ (1992) Demonstration of IHN virus in Germany. Bull Eur Assoc Fish Pathol 12:185-188

Enzmann PJ, Kurath G, Fichtner D, Bergmann SM (2005) Infectious hematopoietic necrosis virus: monophyletic origin of European isolates from North American Genogroup M. Dis Aquat Org 66:187-195

Fauquet CM, Mayo MA, Maniloff J, Desselberger U, Ball LA (eds) (2005) Classification and nomenclature of viruses.

Editorial responsibility: Mark Crane,

Geelong, Victoria, Australia 8th Rep Int Comm Taxon Viruses.Virology Division, International Union of Microbiological Societies. Elsevier Academic Press, Amsterdam

Kurath G, Garver K, Troyer RM, Emmenegger EJ, EinerJensen K, Anderson ED (2003) Phylogeography of infectious haematopoietic necrosis virus in North America. J Gen Virol 84:803-814

Morzunov SP, Winton JR, Nichol ST (1995) The complete genome structure and phylogenetic relationship of infectious hematopoietic necrosis virus. Virus Res 38: 175-192

Pringle CR (1999) Virus taxonomy - 1999. The universal system of virus taxonomy, updated to include the new proposals ratified by the International Committee on Taxonomy of Viruses during 1998. Arch Virol 144:421-429

Schütze H, Enzmann PJ, Kuchling R, Mundt E, Niemann H, Mettenleiter TC (1995) Complete genomic sequence of the fish rhabdovirus infectious haematopoietic necrosis virus. J Gen Virol 76:2519-2527

Submitted: July 2, 2009; Accepted: November 12, 2009

Proofs received from author(s): February 8, 2010 